\title{
Thermal Conductivity, Thermal Diffusivity and Thermoelectric Power of Sm-Based Bulk Superconductors
}

\author{
Hiroyuki Fujishiro and Shuichi Kohayashi
}

\begin{abstract}
The $a b$-plane thermal conductivity $\kappa_{a \dot{b}}(T)$, thermal diffusivity $\alpha_{a b}(T)$ and thermoelectric power $S_{a b}(T)$ were measured for the highly-oriented Ag-doped superconducting materials $\mathrm{Sm}_{1+2 X} \mathrm{Ba}_{2+X} \mathrm{Cu}_{3+X} \mathrm{O}_{7+5 X}(X=0.1 \sim 0.4)$ prepared by a melt process. The absolute value of $\kappa_{a b}$ and its characteristic enhancement below $T_{c}$ were suppressed with increasing content $X$ of the $\mathrm{Sm}_{2} \mathrm{BaCuO}_{5}(\mathrm{Sm} 211)$ secondary phase, while they were enhanced with increasing content of doped-Ag. The application of the magnetic field up to $10 \mathrm{~T}$ clarified that the phonon scattering by vortex was most enhanced with the applied field perpendicular to the $a b$-plane. The trapped magnetic also seriously suppressed the $\kappa_{a b}(T)$ enhancement.
\end{abstract}

Index Terms-Phonon scattering, Sm-based superconductors, thermal conductivity, thermal diffusivity.

\section{INTRÓDUCTION}

$\mathbf{V}$ ARIOUंS kinds of practical applications using the meltprocessed $\mathrm{REBaCuO}$-system superconductors (RE: rare earth ions), such as magnetic bearings, levitation systems, flywheels, bulk magnets, current leads and so on have been recently proposed and developed [1], [2]. The critical current density $J_{c}$ of REBaCuO-system in high magnetic fields has been improved by employing smaller $\mathrm{RE}$ ions such as $\mathrm{Sm}$ and $\mathrm{Nd}$, exceeding those of the $\mathrm{YBaCuO}$-system. The melt-processed RE$\mathrm{BaCuO}$-crystals consist of both the $\mathrm{REBa}_{2} \mathrm{Cu}_{3} \mathrm{O}_{7-\delta}$ (RE123) superconducting phase and the $\mathrm{RE}_{2} \mathrm{BaCuO}_{5}$ (RE211) impurity phase. Silver $(\mathrm{Ag})$ metal is added in order to improve the mechanical strength and to increase $J_{c}$. A small amount of platinum $(\mathrm{Pt})$ metal is also added to disperse finely and uniformly the RE211 particles, which act as pinning centers. For practical applications, thermal properties of superconducting materials such as the thermal conductivity $\kappa$, thermal diffusivity $\alpha$, thermoelectric power $S$, specific heat $C$ are very important. The low thermal conductivity, for example, is essential for the application of bulk superconducting materials as power current leads. We have reported and analyzed the $\kappa$ anisotropy for the bulk Y123 and Y211 composite system [3].

Manusćript received September 24,2001. This work was supported in part by Japan Science and Technology Corporation under the Joint-Research Project for Regional Intensive in the Iwate Prefecture on "Development of practical applications of magnetic field technology for use in the region and in everyday living."

H. Fujishiro is with the Faculty of Engineering, Iwate University, Ueda Morioka 020-8551 Japan (e-mail: fujishiro @iwate-u.ac.jp).

S. Kohayashi is with the Central Research Laboratory, DOWA Mining Co., Ltd., Hachioji 192-0001 Japan (e-mail: kohayass@dowa.co.jp).

Publisher Item Identifier S 1051-8223(02)04138-6.
In the present paper, we report $\kappa_{a b}(T), \alpha_{a b}(T)$ and $S_{a b}(T)$ of the highly-oriented Ag-doped $\mathrm{Sm}_{1+2 X} \mathrm{Ba}_{2+X} \mathrm{Cu}_{3+X} \mathrm{O}_{7+5 X}$ $(X=0.1 \sim 0.4)$ in the temperature range of $10 \sim 300 \mathrm{~K}$ and in the magnetic field of $0 \sim 10 \mathrm{~T}$. We analyze the effect of additions of $\mathrm{Sm}_{2} \mathrm{BaCuO}_{5}(\mathrm{Sm} 211)$ and $\mathrm{Ag}$ metal, on the thermal properties. The magnetic field dependence of $\kappa_{a b}(T)$ is also investigated and the phonon scattering by the vortex is discussed.

\section{EXPERIMENTAL}

The preparation of Ag-doped $\mathrm{Sm}_{1+2 X} \mathrm{Ba}_{2+X} \mathrm{Cu}_{3+X} \mathrm{O}_{7+5 X}$ $(X=0.1,0.2,0.3,0.4)$ bulk superconductors was described elsewhere [4]. The content of the added $\mathrm{Ag}$ was 10 or $15 \mathrm{wt} . \%$ and that of the $\mathrm{Pt}$ was $0.42 \mathrm{wt} \%$ to the $\mathrm{Sm}_{1+2 X} \mathrm{Ba}_{2+X} \mathrm{Cu}_{3+X} \mathrm{O}_{7+5 X}$ weight. The sizes of the $\mathrm{Sm} 211$ and $\mathrm{Ag}$ particles were $2 \sim 5$ and $20 \sim 30 \mu \mathrm{m}$, respectively. From the X-ray diffraction analyses, the grown crystals were crystallographycally highly oriented, where the bulk surface is parallel to the $a b$-plane of Sm123. The slender-shaped samples $(4.0 \mathrm{~mm}$ $\times 4.0 \mathrm{~mm} \times 25.0 \mathrm{~mm}$ ) were cut parallel to the $a b$-plane for the measurement. The $\mathrm{Sm} 211$ polycrystal was also fabricated by the solid state reaction at $1150^{\circ} \mathrm{C}$ in air in order to compare the thermal properties with those of the bulk crystals.

The $a b$-plane thermal conductivity $\kappa_{a b}(T)$ and the thermoelectric power $S_{a b}(T)$ were automatically measured by a steady-state heat flow method between 10 and $300 \mathrm{~K}$ and the thermal diffusivity $\alpha_{a b}(T)$ measurement was performed by an arbitrary heating method under an identical experimental setup with the $\kappa$ and $S$ measurements [5]. A Gifford-McMahon (GM) cycle helium refrigerator was used as a cryostat. The chromel-constantan thermocouples with $\phi 76 \mu \mathrm{m}$ in diameter were used as thermometers for the $\kappa, \alpha$ and $S$ measurements. The magnetic field of up to $10 \mathrm{~T}$ was applied using a cryocooler-cooled superconducting magnet.

\section{RESULTS AND DISCUSSION}

\section{A. Volume Fraction of the Constituent Phases}

The present Ag-doped $\mathrm{SmBaCuO}$ superconductors consist of the Sm123 superconducting phase, the Sm211 insulating phase, and $\mathrm{Ag}$ and Pt particles. The constituent phases and particles contribute the thermal properties of this system. Fig. 1 shows the calculated volume fraction of each component for $\mathrm{Sm}_{1+2 X} \mathrm{Ba}_{2+X} \mathrm{Cu}_{3+X} \mathrm{O}_{7+5 X}(X=0.1 \sim 0.4)$. The measured density and the ideal densities of the Sm123, Sm211, Ag and Pt $\left(6.87,7.46,10.25\right.$ and $21.37 \mathrm{~g} / \mathrm{cm}^{3}$, respectively) were used 


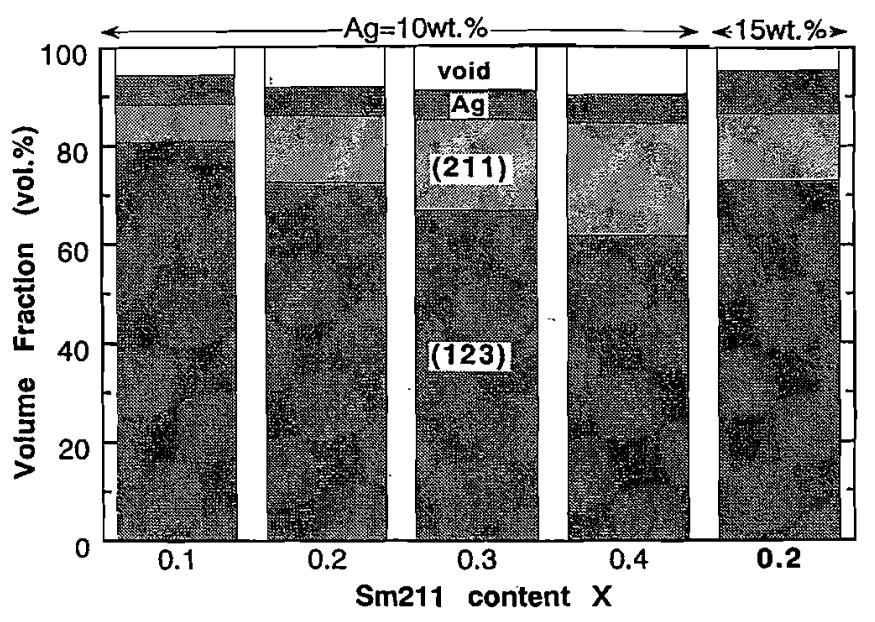

Fig. 1. The volume fraction of each component for $\mathrm{Sm}_{1+2 X} \mathrm{Ba}_{2+X} \mathrm{Cu}_{3+X} \mathrm{O}_{7+5 X}(X=0.1 \sim 0.4)$ for $\mathrm{Ag}=10$ wt. $\%$ and 15 wt. $\%(X=0.2)$ samples.

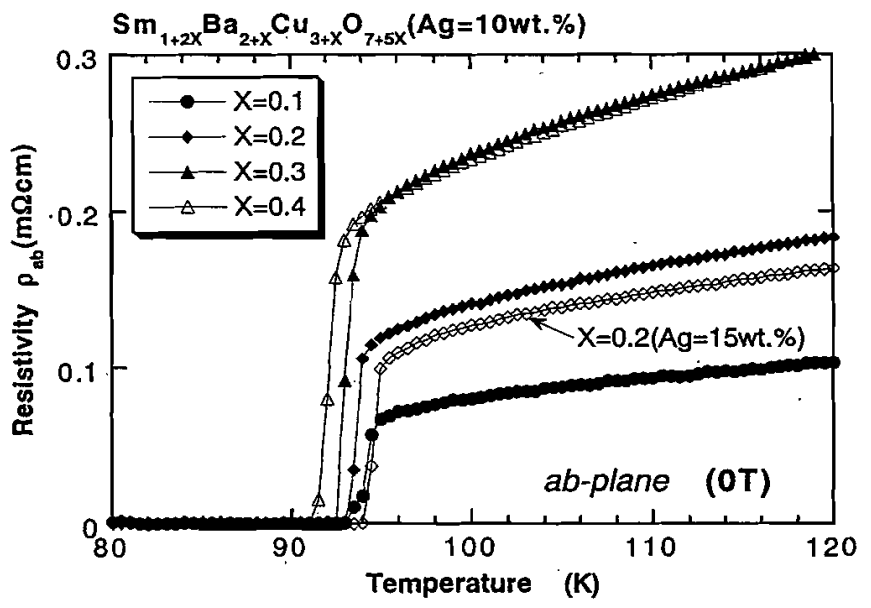

Fig. 2. The temperature dependence of the $a b$-plane resistivity $\rho_{a b}(T)$.

for the calculation. The volume fraction of $\mathrm{Pt}$ was omitted in the figure because it is very small $(<0.12$ vol. $\%)$. The volume fraction of $\operatorname{Sm} 123$ decreases and that of $\operatorname{Sm} 211$ increases with increasing $X$. It should be noticed that the fraction of void also increases with increasing $X$. In Fig. 1, the volume fractions are also shown for the $\mathrm{Ag}=15 \mathrm{wt} . \%(X=0.2)$ sample. The increase of the Ag content to $15 \mathrm{wt} . \%$ markedly reduces voids possibly through the intrusion of $\mathrm{Ag}$ particles into the grain boundaries.

Fig. 2 shows the temperature dependence of the $a b$-plane electrical resistivity $\rho_{a b}(T)$. The $\rho_{a b}$ values for the $\mathrm{Ag}=10$ wt.\% samples increase with increasing $X$, which may result from the increase of the insulating $\operatorname{Sm} 211$ fraction. The superconducting transition temperature $T_{c}$ slightly decreases with increasing $X$. As can be seen for the $X=0.2$ sample, the $\rho_{a b}(T)$ decreases by increasing the $\mathrm{Ag}$ content from 10 to $15 \mathrm{wt}$.\%. For the $X=0.2$ and $\mathrm{Ag}=15$ wt.\% sample, $T_{c}$ recovers to $94 \mathrm{~K}$, the maximum value of the present samples. The $X$ dependence and the Ag content dependence of $T_{c}$ cannot be interpreted by only the changes of the volume fraction of the $\mathrm{Sm} 123$ phase. It is suggested that the superconductivity of the $\mathrm{Sm} 123$ phase is improved by the increase of $\mathrm{Ag}$ content up to $15 \mathrm{wt} . \%$.

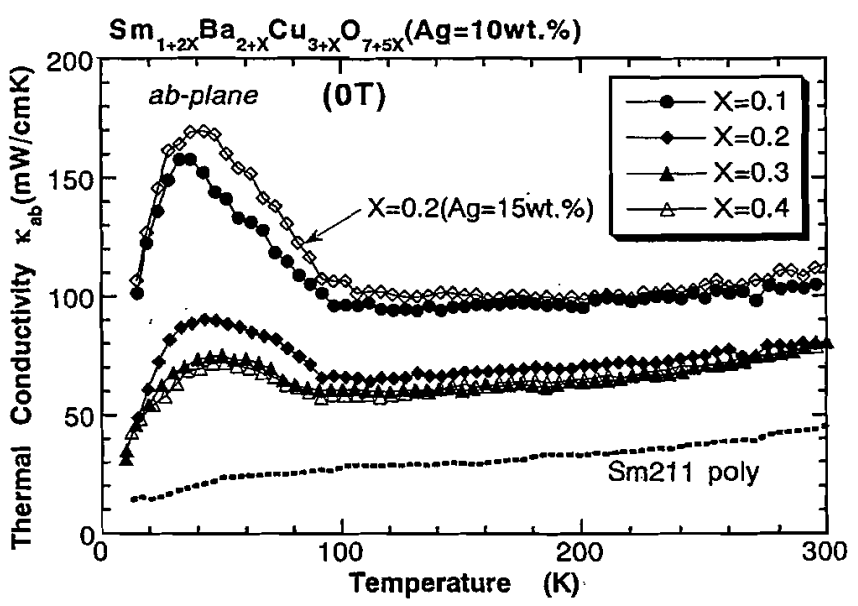

Fig. 3. The temperature dependence of the $a b$-plane thermal conductivity $\kappa_{a b}(T)$ for various $X$ under the zero magnetic field.

\section{B. Thermal Conduction in Zero Magnetic Field}

Fig. 3 shows the temperature dependence of $\kappa_{a b}(T)$ for various $X$ with $\mathrm{Ag}=10 \mathrm{wt} . \%$. For the $X=0.1$ sample $\cdot$ which consists of the maximum amount of $\mathrm{Sm} 123$ superconducting phase, the absolute $\kappa_{a b}$ value above $T_{c}(=93 \mathrm{~K})$ is large and the $\kappa_{a b}(T)$ enhancement below $T_{c}$ is prominent. For the $X=0.2$ sample, the $\kappa_{a b}(T)$ enhancement becomes less prominent and it is further reduced with further increase of $X$. At the same time, $\kappa_{a b}(T)$ in the normal state decreases. However, it is to be noticed that $\kappa_{a b}(T)$ values of $X=0.3$ and $X=0.4$ samples almost coincide each other over the entire temperature range. $\kappa(T)$ of the $\operatorname{Sm} 211$ polycrystals is also presented in Fig. 3, which decreases with decreasing temperature and is very small, making a contrast with that of the Y211 polycrystal [3]. $\kappa(T)$ of Y211 was somewhat larger, took a faint maximum at $T \sim 50 \mathrm{~K}$ and decreased with further increase of $T$. The electronic thermal conductivity $\kappa_{e}(T)$, estimated by the Wiedemann-Franz law using the $\rho_{a b}$ values, decreases with increasing $X$. The ratio of $\kappa_{e}$ to the total $\kappa$ at $300 \mathrm{~K}$ is $31 \%$ and $16 \%$ for $X=0.1$ and 0.4 , respectively. The decrease of $\kappa_{a b}(T)$ with increasing $X$ above $T_{c}$ may come mainly from the reduction of $\kappa_{e}$, the increase of low thermal conductive $\operatorname{Sm} 211$ phase and the enhanced phonon scattering in the Sm123 phase.

It has been found that in $\mathrm{REBaCuO}$-systems with the light RE (LRE) element such as Sm and Nd, a part of the LRE ions is substituted for the $\mathrm{Ba}$ site, thus forming the $\mathrm{LRE}_{1+y} \mathrm{Ba}_{2-y} \mathrm{Cu}_{3} \mathrm{O}_{z}$-type solid solution [1]. Then $T_{c}$ should decrease with increasing $\mathrm{RE}$ ion substitution. $T_{c}$ actually decreases with increasing $X$ as shown in Fig. 2, which suggests increased substitution of $\mathrm{Sm}$ for $\mathrm{Ba}$ site with increasing $X$. The increased migration of $\mathrm{Sm}$ and $\mathrm{Ba}$ enhances the phonon scattering in the Sm123 phase, working as a kind of point defect type phonon scattering centers.

The thermal conductivity of high $T_{c}$ oxide superconductors (HTSC) is enhanced below $T_{c}$ by both the electronic contribution $\kappa_{e}$ and the phonon contribution $\kappa_{p h}$. In not ideally pure HTSC with sizable residual electron scattering by impurities, the enhancement mainly comes from the phonon component $\kappa_{p h}$ [6]. The $\kappa_{p h}$ enhancement below $T_{c}$ is caused by the reduction in the phonon scattering by quasiparticles with the 


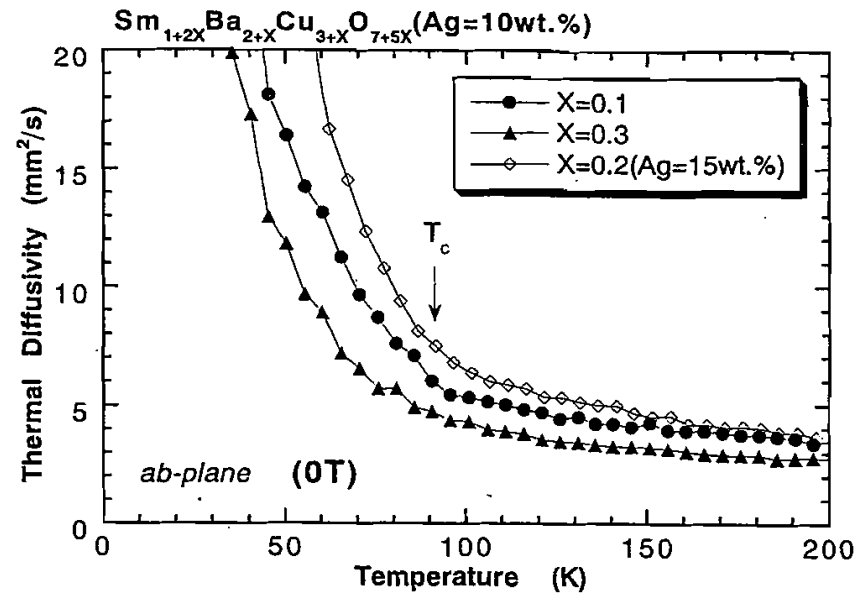

Fig. 4. The temperature dependence of the $a b$-plane thermal diffusivity $\alpha_{a b}(T)$ for typical samples.

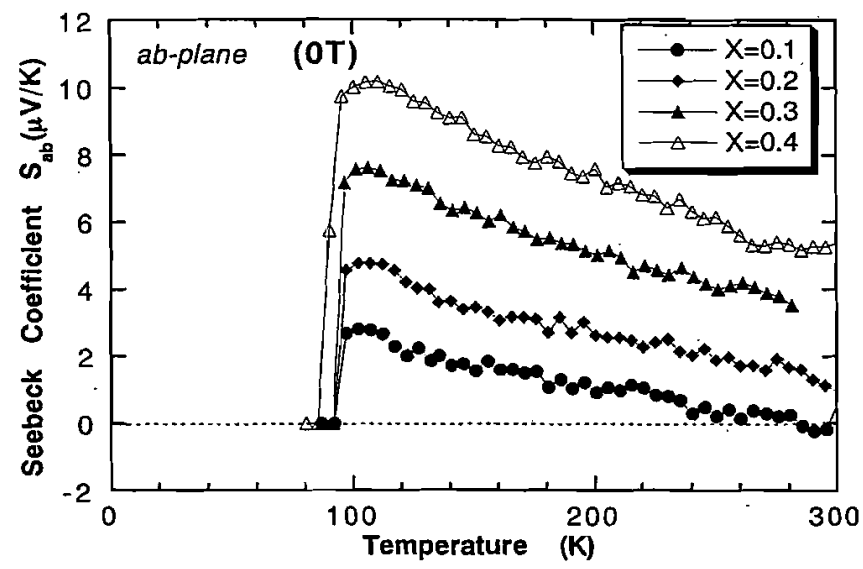

Fig. 5. The temperature dependence of the thermoelectric power $S_{a b}(T)$ in the $a b$-plane.

progress of their condensation into cooper pairs [5]. Because the present $\mathrm{Sm}_{1+2 X} \mathrm{Ba}_{2+X} \mathrm{Cu}_{3+X} \mathrm{O}_{7+5 X}$ samples belong to the impure HTSC material containing a considerable amount of the Sm and $\mathrm{Ba}$ migration and the impurity $\mathrm{Sm} 211$ phase, the $\kappa$ enhancement is due to the $\kappa_{p h}$ contribution. The $\kappa_{p h}$ enhancement is masked out if the phonon scattering strength by other mechanisms becomes stronger. The suppression of the $\kappa$ enhancement with increasing $X$ may reflect the enhanced phonon scattering due to increased $\mathrm{Sm}$ and $\mathrm{Ba}$ migration. For the $X=0.2$ sample with $\mathrm{Ag}=15 \mathrm{wt} . \%, T_{c}$ recovers to $94 \mathrm{~K}$ and then the absolute value and the $\kappa_{a b}(T)$ enhancement also recover as shown in Fig. 3.'This may result from the suppression of the migration and/or from the improved crystallinity in the $\operatorname{Sm} 123$ phase.

Fig. 4 shows the temperature dependence of $\alpha_{a b}(T)$. In the normal state, $\alpha_{a b}(T)$ of all the samples increases slightly with decreasing temperature. Below $T_{c}, \alpha_{a b}(T)$ increases more and more rapidly with decreasing temperature. The absolute value of $\alpha$ decreases with the increase of $X$ and increases with increasing content of the doped-Ag, in accord with the behavior of $\kappa_{a b}$.

Fig. 5 shows the temperature dependence of $S_{a b}(T)$. For the $\mathrm{Ag}=10 \mathrm{wt} . \%$ samples, $S_{a b}(T)$ above $T_{c}$ positively increases
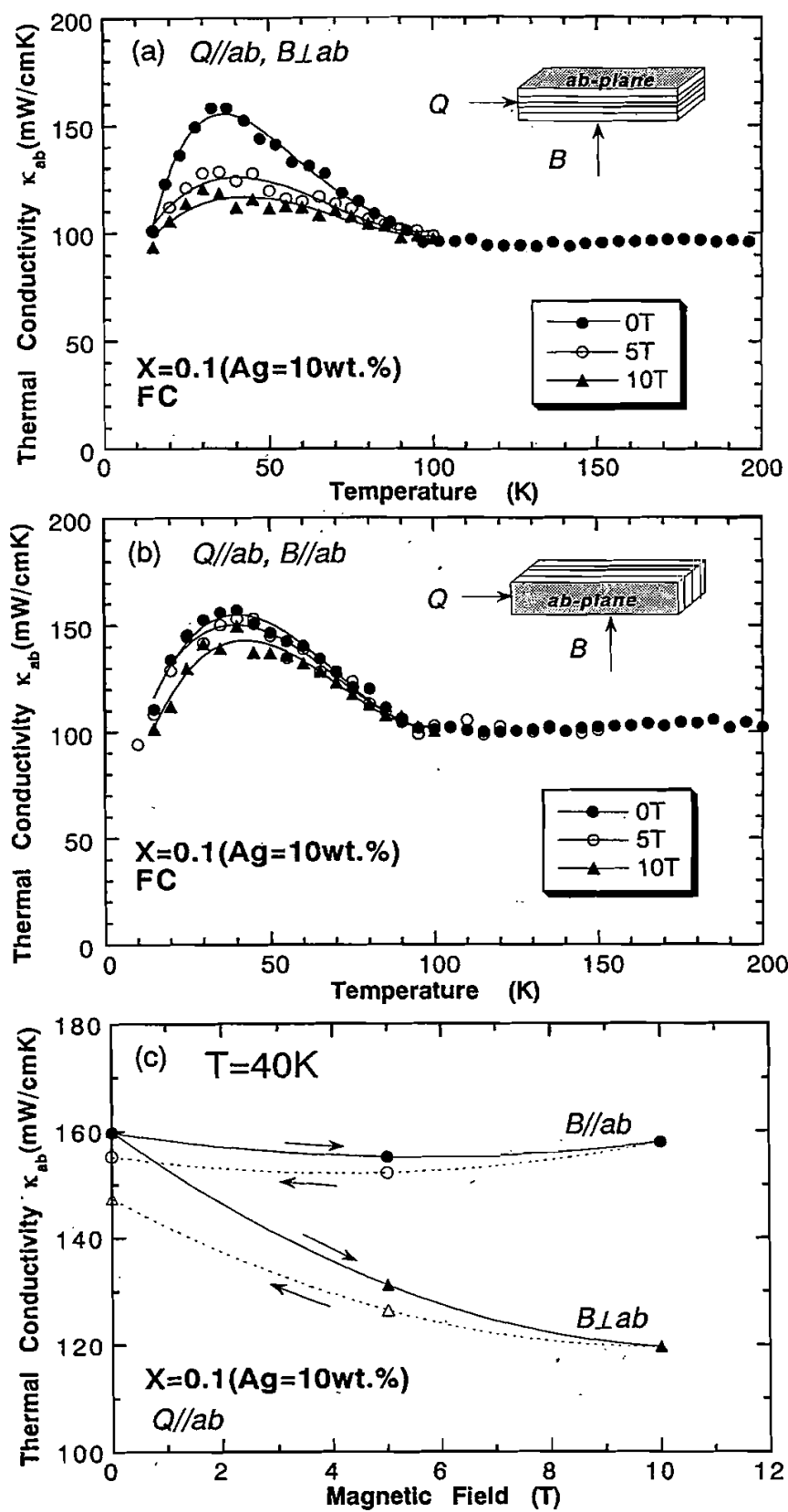

Fig. 6. The $a b$-plane thermal diffusivities $\kappa_{a b}(T)$ with the applied field $B$ (a) perpendicular and (b) parallel to the $a b$-plane, respectively, for the $X=0.1$ $(\mathrm{Ag}=10 \mathrm{wt} . \%)$ sample. (c) The magnetic field dependence of $\kappa_{a b}$ at $40 \mathrm{~K}$ where $B$ in both parallel and perpendicular directions to the $a b$-plane.

with increasing $X$. The thermoelectric power $S$ of a metal is given by

$$
S=\frac{\pi^{2}}{3}\left(\frac{k}{e}\right) k T \rho \frac{\partial}{\partial E}\left(\frac{1}{\rho(E)}\right)
$$

where $E$ the electron energy. Quantitatively, $S$ is expected to increase in impure metals because of increasing $\rho$ values. Accordingly, the observed increasing $S_{a b}$ with $X$ may partly be attributable to the $\rho_{a b}(T)$ increase in the $S m 123$ phase caused by the migration effect. Since the present Ag-doped Sm-based material is a composite system composing of $\mathrm{Ag}$ particle, $\mathrm{Sm} 123$ and $\operatorname{Sm} 211$, it is difficult to analyze $S_{a b}$. However, it is worth 
while to note that $S_{a b}$ of the $X=0.4$ sample is clearly larger than that of the $X=0.3$ sample in spite of almost indistinguishable $\rho_{a b}$. This fact indicates that the Sm211 phase also intrinsically, i.e., not only through the increase of $\rho_{a b}$, enhances $S_{a b}$ of this composite system.

\section{Thermal Conduction Under the Magnetic Field}

In this subsection, we present $\kappa_{a b}$ of the $X=0.1(\mathrm{Ag}=10$ wt.\%) sample under the magnetic fields. Fig. 6(a) and (b) shows $\kappa_{a b}$ for field cooling runs with the applied field $B$ perpendicular and parallel to the $a b$-plane, respectively. The solid lines are to guide the reader's eyes. In both figures, $\kappa_{a b}$ above $T_{c}$ is independent of the applied magnetic fields. In Fig. $6(a)$, the $\kappa_{a b}$ enhancement below $T_{c}$ is drastically suppressed with increasing $B$ perpendicular to the $a b$-plane. This reduction can be understood as caused by the enhanced phonon scattering by the quasiparticles in the vortex cores. For the application of $B$ parallel to the $a b$-plane, however, the reduction of the $\kappa_{a b}$ enhancement is barely discernible as shown in Fig. 6(b). In this case, the vortices parallel to the $a b$-plane hardly hinders the heat flow. The similar anisotropic effect of the magnetic field on $\kappa_{a b}(T)$ was observed for YBCO and Bi-2212 single crystals.

Fig. 6(c) shows the magnetic field dependence of $\kappa_{a b}$ at $40 \mathrm{~K}$ where $B$ was swept up and down up to $10 \mathrm{~T}$. For the $B \perp a b$-plane setting, $\kappa_{a b}(40 \mathrm{~K})$ promptly decreases with increasing magnetic field and partly recovers with decreasing field; very clear hysteretic behavior of $\kappa_{a b}$ is noticed. The $\kappa_{a b}(40 \mathrm{~K})$ value at $0 \mathrm{~T}$ after applying field is $8 \%$ smaller than the initial one. This suggests that the trapped magnetic field scatters the phonon transport even after removing the field. The trapped magnetic field for the $B \perp a b$-plane setting is estimated to be about $2 \mathrm{~T}$ from the data for the increasing virgin run. On the other hand, for the $B / / a b$-plane setting, the field dependence of $\kappa_{a b}(40 \mathrm{~K})$ are quite small up to the applied field of $10 \mathrm{~T}$.

Lastly, we comment on the effect of the added-Ag particles on the thermal transport. In the previous paper, we reported the thermal conductivity of the $\mathrm{Ag}+\mathrm{YBCO}$ mixed crystals for various $\mathrm{Ag}$ contents and determined the purity of $\mathrm{Ag}$ particles in these crystals [7]. Using the previous data, the residual resistivity ratio (RRR) of the doped-Ag particles is estimated to be $\sim 15$ in the present case. The measured thermal conductivity of $\mathrm{Ag}+0.22$ at.\% Au alloy with RRR $\sim 15$ does not show the $\kappa(T)$ enhancement around $40 \mathrm{~K}$. The addition of 10 and $15 \mathrm{wt} \% \mathrm{Ag}$ enhances the absolute value of $\kappa(T)$ over the entire temperature range, but it cannot directly cause the $\kappa_{a b}(T)$ enhancement below $T_{c}$.

\section{SUMMARY}

The $a b$-plane thermal conductivity $\kappa_{a b}(T)$, diffusivity $\alpha_{a b}(T)$ and thermoelectric power $S_{a b}(T)$ were measured for highly oriented Ag-doped $\mathrm{Sm}_{1+2 X} \mathrm{Ba}_{2+X} \mathrm{Cu}_{3+X} \mathrm{O}_{7+5 X}$ materials, which consisted of superconducting $\mathrm{Sm}_{1} \mathrm{Ba}_{2} \mathrm{Cu}_{3} \mathrm{O}_{7}$. (Sm123) and semiconductive $\mathrm{Sm}_{2} \mathrm{Ba}_{1} \mathrm{Cu}_{1} \mathrm{O}_{5}$ (Sm211) phases by the ratio $1: X$. The obtained results are summarized as follows. i) For the $\mathrm{Ag}=10 \mathrm{wt} . \%$-doped series, the $\kappa_{a b}(T)$ values and the $\kappa_{a b}$ enhancement below $T_{c}$, characteristic of high- $T_{c}$ superconductors, are largest for $X=0.1$, which are rapidly reduced with increasing $X$ up to $X=0.3 . \kappa_{a b}(T)$ values became almost identical for the $X=0.3$ and $X=0.4$ samples. An important origin for the reduction was suggested to come from increasing migration of $\mathrm{Sm}$ and $\mathrm{Ba}$ ions. The quality of the $\operatorname{Sm} 123$ crystal was deteriorated with decreasing $X$, as suggested also by the $T_{c}$ dependence on $X$. The increasing Ag-doping from 10 wt.\% to $15 \mathrm{wt} . \%$ for the $X=0.2$ crystal enhanced the $\kappa_{a b}(T)$ values, accompanying the $T_{c}$ increase to $94 \mathrm{~K}$. These results indicate that the deteriorated crystallinity of $\operatorname{Sm} 123$ is recovered or improved by the increased Ag-doping. The diffusivity $\alpha_{a b}(\mathcal{T})$ behaved consistently to $\kappa_{a b}$.

ii) Under the magnetic field up to $10 \mathrm{~T}$, the $\kappa_{a b}$ enhancement was markedly reduced for the applied field configuration, $B \perp a b$-plane. In contrast, very weak suppression was observed for the $B / / a b$-plane configuration. These results are consistent with the explanation that the $\kappa_{a b}$ enhancement below $T_{c}$ originates from the reduction in the phonon scattering by quasiparticles. The anisotropic suppression with respect to the field direction can be understood to come from the appearance of anisotropic vortex scattering for phonons which originates from the layer structure of Sm123 lattice. From the hysteretic behavior of $\kappa_{a b}(B)$ under the applied fields, we could estimate the trapped magnetic flux $B_{r e s}$ to be about $2 \mathrm{~T}$ after the 10 $T$ field scan in the $B \perp a b$-plane configuration.

iii) The $a b$-plane thermoelectric power $S_{a b}$ was positive and increased with increasing $X$, precisely reflecting the $\operatorname{Sm} 211$ content $X$ up to $X=0.4$. It is worthwhile to notice that both $\rho_{a b}(T)$ and $\kappa_{a b}(T)$ behaved almost identically for $X=0.3$ and 0.4 samples, making a sharp contrast with the behavior of $S_{a b}$.

\section{REFERENCES}

[1] M. Murakami, N. Sakai, T. Higuchi, and S. I. Yoo, "Melt-processed light rare earth element-Ba-Cu-O," Supercond. Sci. Technol., vol. 9, pp. 1015-1032, 1996.

[2] M. Murakami, Melt Processed High-Temperature Superconductors. Singapore: World Scientific, 1993.

[3] H. Fujishiro, M. Ikebe, T. Naito, K. Noto, S. Kohayashi, and S. Yoshizawa, "Anisotropic thermal diffusivity and conductivity of YBCO(123) and YBCO (211) mixed crystal I,"Jpn. J. Appl. Phys., vol. 33, pp. $4965-4970,1994$

[4] S. Kohayashi and S. Nagaya, "Crystal structure and magnetic properties of melt-processed $\mathrm{Sm}_{1+2 x} \mathrm{Ba}_{2+x} \mathrm{Cu}_{3+x} \mathrm{O}_{y}$," Phys. C, submitted for publication.

[5] M. Ikebe, H. Fujishiro, T. Naito, and K. Noto, "Simultaneous measurement of thermal diffusivity and conductivity applied to $\mathrm{Bi}-2223$ ceramic superconductors," J. Phys. Soc. Jpn., vol. 63, pp. 3107-3114, 1995.

[6] M. Ikebe, H. Fujishiro, K. Nakasato, T. Mikami, T. Naito, and T. Fukase, "Thermal transport in $90 \mathrm{~K}$ - and $60 \mathrm{~K}$-phase $\mathrm{YBa}_{2} \mathrm{Cu}_{3} \mathrm{O}_{7-\delta}$ oxides," Phys. Stat. Sol. (b), vol. 209, pp. 413-426, 1998.

[7] H. Fujishiro, M. Ikebe, Y. Minato, H. Ogasawara, and K. Noto, "Model analyses of thermal conductivity and purity of doped $\mathrm{Ag}$ in $\mathrm{Ag}+\mathrm{YBa}_{2} \mathrm{Cu}_{3} \mathrm{O}_{7}$," Superlattice \& Microstructure, vol. 21, pp. 349-352, 1997. 